\title{
On the effect of the molecular diffusivity in turbulent diffusion
}

\author{
By P. G. SAFFMAN \\ Department of Applied Mathematics and Theoretical Physics, \\ University of Cambridge
}

(Received 3 October 1959)

It is shown that the dispersion of a substance, with molecular diffusivity $\kappa$, in a stationary, homogeneous, turbulent velocity field can be formulated in terms of a 'substance auto-correlation function', this being a generalization of the wellknown Lagrangian correlation between the velocity of a fluid particle at different times. It is found that the interaction between the molecular diffusion and the turbulent motion reduces the dispersion from the value it would have if the processes of molecular and turbulent diffusion were independent and additive. The conflict, between the results obtained in this paper and previous results which implied that the interaction increases the dispersion, is resolved. The ratio, of the contributions to the dispersion from the interaction term and the turbulent diffusion term, is obtained for comparatively large times by the use of intuitive arguments, and is found to be inversely proportional to the Prandtl number and a Reynolds number of the turbulence.

\section{Introduction}

Taylor (1921) showed that the mean-square displacement or dispersion, in a given direction, of infinitesimal fluid particles from their original position in a turbulent velocity field is

$$
\overline{Y^{2}(t)}=2 \int_{t_{0}}^{t} d t^{\prime} \int_{t_{0}}^{t^{\prime}} \overline{v\left(t^{\prime}\right) v\left(t^{\prime \prime}\right)} d t^{\prime \prime}
$$

where $v(t)$ is the velocity of a fluid element or particle in the given direction. The integrand

$$
\overline{v\left(t^{\prime}\right) v\left(t^{\prime \prime}\right)}=R_{p}\left(t^{\prime}, t^{\prime \prime}\right), \text { say, }
$$

is the covariance of the velocity of a fluid element at time $t^{\prime \prime}$ and later time $t^{\prime}$, and is a Lagrangian auto-correlation function. When the velocity of a fluid element is statistically stationary in time,

and

$$
\begin{gathered}
R_{p}\left(t^{\prime}, t^{\prime \prime}\right)=\overline{v^{2}} S_{p}\left(t^{\prime}-t^{\prime \prime}\right) \\
\overline{Y^{2}}=2 \overline{v^{2}} \int_{0}^{t-t_{0}}\left(t-t_{0}-\tau\right) S_{p}(\tau) d \tau
\end{gathered}
$$

(see the review article by Batchelor \& Townsend (1956); this is referred to hereafter as I). 
When the quantity or substance being dispersed is subject to molecular diffusion (e.g. mass or heat) with a diffusivity $\kappa$, it can be shown (see I) that, provided $t-t_{0}$ is sufficiently small that

$$
t-t_{0} \ll\left(\overline{\omega^{2}}\right)^{-\frac{1}{2}}, \quad \frac{\kappa}{\nu}\left(t-t_{0}\right) \ll\left(\overline{\omega^{2}}\right)^{-\frac{1}{2}},
$$

the effects of turbulent and molecular diffusion are linearly additive in the first approximation, giving a total dispersion

$$
D^{2}=\overline{Y^{2}}+2 \kappa\left(t-t_{0}\right),
$$

where $\omega$ is the vorticity of the turbulent motion and $\nu$ is the kinematic viscosity. (Henceforth, for brevity, we shall write $\omega=\left(\overline{\omega^{2}}\right)^{\frac{1}{2}}$.) For larger values of $t-t_{0}$, there is an interaction between the turbulent and molecular diffusion. Arguments are presented in I (due originally to Townsend 1954) which give the improved approximation

$$
\Delta=D^{2}-\overline{Y^{2}}-2 \kappa\left(t-t_{0}\right)=\frac{28}{45} \kappa \omega^{2}\left(t-t_{0}\right)^{3}
$$

to $O\left(t-t_{0}\right)^{3}$ for times such that $(1.5)$ is satisfied. That is, the effect of the interaction is initially, and by implication for later times, to increase the dispersion over and above that due to the turbulent and molecular diffusions acting independently.

It is the purpose of the present note to show that there is a fallacious step in the deduction of $(1,7)$, and that in fact for stationary homogeneous turbulence

$$
\Delta=-\frac{1}{9} \kappa \omega^{2}\left(t-t_{0}\right)^{3}
$$

to $O\left(t-t_{0}\right)^{3}$ when (1.5) is satisfied. Intuitive arguments are also given to show that, for larger values of $t-t_{0}, \Delta$ is a decreasing function of $t-t_{0}$ and inversely proportional to the Prandtl number $\nu / \kappa$. This is in qualitative agreement with measurements by Mickelsen (1960) of the width of the diffusion wake behind a source emitting helium $\left(\kappa=0.725 \mathrm{~cm}^{2} / \mathrm{s}\right)$ and carbon dioxide $\left(\kappa=0.167 \mathrm{~cm}^{2} / \mathrm{s}\right)$ in the turbulent air stream downstream of a grid in a wind tunnel. He found for values of $t-t_{0}$ comparable with a time characteristic of the energy containing eddies that the values of $D^{2}-2 \kappa\left(t-t_{0}\right)$ were $5 \%$ or more smaller for helium than for carbon dioxide.

\section{The substance auto-correlation coefficient}

The reason why the dispersion of a substance differs from that of infinitesimal fluid particles is because the molecules of the substance do not move with the velocity of the fluid continuum (which can be regarded as a continuous function of position and time), but have relative to this velocity a random thermal or Brownian velocity, whereas the infinitesimal fluid particles are points which by definition move with the local fluid velocity. When the fluid velocity is zero, this Brownian velocity manifests itself as the molecular diffusivity.

The dispersion of a mass-like substance released from a source in a turbulent flow (e.g. a gas in a turbulent air stream) is the ensemble or stochastic average of the displacement (squared) of a marked molecule of the substance, where the average is over the ensemble of all Brownian motions and the ensemble of all 
realizations of the turbulent velocity field. The velocity of the molecule in a given direction is $V(t)+q(t)$, where $V(t)$ is the velocity of the fluid at the point occupiod by the molecule and $q(t)$ is the Brownian velocity. Hence, by analogy with (1.1),

$$
D^{2}(t)=2 \int_{t_{0}}^{t} d t^{\prime} \int_{t_{0}}^{t^{\prime}} \Re\left(t^{\prime}, t^{\prime \prime}\right) d t^{\prime \prime}
$$

where

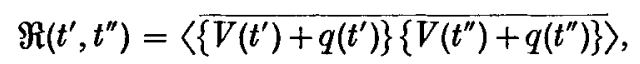

the angle brackets denoting an average over the Brownian ensemble and the overbar an average over the turbulent ensemble, and $V(t)+q(t)$ is the total (component of) velocity at time $t$ of a marked molecule released from the source, at $\mathbf{x}_{0}$ say, at time $t_{0}$.

In the absence of fluid velocity, in a homogeneous medium, $q(t)$ is a stationary random function of $t$ with zero mean, so that

$$
\left\langle q\left(t^{\prime}\right) q\left(t^{\prime \prime}\right)\right\rangle=Q\left(t^{\prime}-t^{\prime \prime}\right)
$$

and since in this case $D^{2}=2 \kappa\left(t-t_{0}\right)$, it follows (see (1.3) and (1.4)) that

$$
\int_{0}^{\infty} Q(\tau) d \tau=\kappa
$$

where $\kappa$ is the molecular diffusivity. Moreover, it is known that $Q(\tau)$ is negligible when $\tau \gg \tau_{c}$, where $\tau_{c}$ is the mean time between molecular collisions, so that (2.4) holds when the upper limit of integration is any time large compared with $\tau_{c}$.

We now suppose that the ambient fluid is in turbulent motion, and in particular we shall assume that the turbulence is statistically stationary and homogeneous*. In this case, $q(t)+V(t)$ is a stationary random function of $t$ with zero mean, and $\Re$ is a function of $t^{\prime}-t^{\prime \prime}$, i.e.

$$
\begin{aligned}
\Re\left(t^{\prime}, t^{\prime \prime}\right) & =\overline{\langle\{V(t)+q(t)\}\{V(t+\tau)+q(t+\tau)\}}\rangle \\
& =\subseteq(\tau), \quad \text { say, }
\end{aligned}
$$

where $\tau=t^{\prime}-t^{\prime \prime}$. Then $D^{2}$ is given by $(1 \cdot 4)$ with $\overline{v^{2}} S_{p}$ replaced by $\Im$.

Now the statistical distribution of $q(t)$ is determined by the molecular collisions with a time scale $\tau_{c}$, whereas the time scale of the macroscopic motion is at least of order $\omega^{-1}$. Now $\omega \tau_{c} \ll 1$, since otherwise it is not meaningful to regard the fluid as a continuum, and since the two time scales are so different, the Brownian motion and the macroscopic motion will be statistically uncorrelated (for a fuller discussion of this point see the Appendix). Hence, with error of order $\omega \tau_{c}$,

$$
\begin{aligned}
& \circlearrowleft(\tau)=\langle\overline{V(t) V(t+\tau)}\rangle+\overline{\langle q(t) q(t+\tau)}\rangle \\
& =\bar{v}^{2} S_{d}(\tau)+Q(\tau), \quad \text { say, }
\end{aligned}
$$

where $Q(\tau)$ has the value it would have in the absence of fluid motion, and $\overline{v^{2}}$ is the mean square of a component of the turbulent velocity. It now follows that

$$
D^{2}=2 \overline{v^{2}} \int_{0}^{t-t_{0}}\left(t-t_{0}-\tau\right) S_{d}(\tau) d \tau+2 \kappa\left(t-t_{0}\right)
$$

* It can be shown that the analysis given below is valid if the turbulence is homogeneous in the direction of the dispersion only (e.g. longitudinal dispersion in a pipe). However, for the sake of simplicity of exposition, we restrict the discussion to completely homogeneous turbulence. 
and it can be shown (see the Appendix) that the error in (2.7) is of order $\kappa \omega \tau_{c}\left(t-t_{0}\right) . S_{d}(\tau)$ may be called the 'substance auto-correlation coefficient'.

Note that $S_{d}(\tau) \neq S_{p}(\tau)$, since the former is the correlation coefficient of the fluid velocity at two points which lie on the trajectory of a molecule, and the latter is the correlation at two points on the trajectory of an infinitesimal fluid particle.

An expression for $S_{d}(\tau)$ can be obtained as follows. Let $\theta\left(\mathbf{x}, t \mid \mathbf{x}_{0}, t_{0}\right)$ denote the solution of

$$
\left.\begin{array}{c}
\frac{\partial \theta}{\partial t}+\mathbf{u} \cdot \nabla \theta=\kappa \nabla^{2} \theta, \\
\theta\left(\mathbf{x}, t_{0} \mid \mathbf{x}_{0}, t_{\mathbf{0}}\right)=\delta\left(\mathbf{x}-\mathbf{x}_{\mathbf{0}}\right) .
\end{array}\right\}
$$

(It is assumed throughout that the substance is dynamically passive so that the distribution of $\theta$ has no effect on the turbulent velocity field $\mathbf{u}(\mathbf{x}, t)$.) Then $\theta d \mathbf{x}$ is the probability, for one realization of the turbulence, that a marked molecule is in the element of volume $d \mathbf{x}$ at time $t$, given that it was at $\mathbf{x}_{0}$ at time $t_{0}$. Let $u_{2}(\mathbf{x}, t)$ be the turbulent velocity component in the same direction as $V$. Then $V\left(t_{0}\right)=u_{2}\left(\mathbf{x}_{0}, t_{0}\right)$, and

so that

$$
\begin{gathered}
\langle V(t)\rangle=\int u_{2}(\mathbf{x}, t) \theta\left(\mathbf{x}, t \mid \mathbf{x}_{0}, t_{0}\right) d \mathbf{x}, \\
\overline{v^{2}} S_{d}(\tau)=\left\langle\overline{V(t) \overline{\left.V\left(t_{0}\right)\right\rangle}}\right. \\
=\int \overline{u_{2}(\mathbf{x}, t) u_{2}\left(\mathbf{x}_{0}, t_{0}\right)} \overline{\theta\left(\mathbf{x}, t \mid \overline{\mathbf{x}_{0}, t_{0}}\right)} d \mathbf{x},
\end{gathered}
$$

where $t=t_{0}+\tau$. In the next section, we shall calculate $S_{d}(\tau)$ for small $\tau$, and in $\S 5$ obtained an estimate of its value for large $\tau$.

Incidentally, to put $\kappa=0$ is equivalent to saying that $q \equiv 0$ and that a molecule and an infinitesimal fluid particle always coincide. In this case, $V(t)=v(t)$, where $v(t)$ refers to the velocity of a fluid particle (in contrast to $V(t)$ which is the velocity of the fluid following a molecule), and $S_{d}(\tau)=S_{p}(\tau)$.

The expression (2.7) for $D^{2}$ involves only the fluid velocity and the molecular diffusivity $\kappa$, and should presumably describe the dispersion of a non mass-like quantity like heat, whose diffusion is not readily identifiable with the dispersion of a marked molecule. This could be verified if it were possible to derive (2.7) and (2.10) from formal manipulation of the diffusion equation (2.8) and the expression

$$
D^{2}=\int x_{2}^{2} \theta d \mathbf{x}
$$

but an argument given in the Appendix implies that this may not be possible.

\section{Calculation of $S_{d}(\tau)$ for small $\tau$}

When the second of the conditions (1.5) is satisfied, the size of an element of substance released at $\mathbf{x}_{0}, t_{0}$ is small compared with the length scale of the rotational and straining motions of the turbulence. The diffusion equation (2.1) for $\theta\left(\mathbf{x}, t \mid \mathbf{x}_{\mathbf{0}}, t_{\mathbf{0}}\right)$ can then be written approximately as

$$
\frac{\partial \theta}{\partial t}+x_{j}^{\prime} \frac{\partial \theta}{\partial x_{i}^{\prime}}\left(\frac{\partial u_{i}}{\partial x_{j}}\right)_{p}=\kappa \nabla^{\prime 2} \theta
$$


where $\mathbf{x}^{\prime}$ is the position vector relative to the fluid particle with which the element of substance originally coincided, and the suffix $p$ denotes that quantities are evaluated at the fluid particle (and are therefore functions of $t$ only). It is easily verified that a solution of (3.1) exists of the form

$$
\theta=\theta_{m}(t) \exp \left\{-\frac{1}{2} \alpha_{i j} x_{i}^{\prime} x_{j}^{\prime}\right\}
$$

(see Townsend 1951), and that

$$
\alpha_{i j}=\left\{\kappa\left(t-t_{0}\right)\right\}^{-1}\left[\delta_{i j}+O\left\{\omega\left(t-t_{0}\right)\right\}\right] .
$$

To evaluate $S_{d}$, we note that

$$
u_{2}(\mathbf{x}, t)=v(t)+x_{j}^{\prime}\left(\frac{\partial u_{2}}{\partial x_{j}}\right)_{p}+\frac{1}{2} x_{j}^{\prime} x_{k}^{\prime}\left(\frac{\partial^{2} u_{2}}{\partial x_{j} \partial x_{k}}\right)_{p}+O\left(x^{\prime 3} \frac{\partial^{3} u_{2}}{\partial x^{3}}\right)
$$

where $v(t)$ is the (component of) velocity of the fluid particle which was at $\mathbf{x}_{0}$ at time $t_{0}$. Substituting into (2.9) and making use of (3.2), (3.3) and (3.4), we obtain

$$
\langle V(t)\rangle=v(t)+\kappa\left(t-t_{0}\right)\left(\nabla^{2} u_{2}\right)_{p}\left[1+O\left\{\omega\left(t-t_{0}\right)\right\}\right] .
$$

Hence, from $(2.10)$ and the fact that $V\left(t_{0}\right)=v\left(t_{0}\right)$,

$$
\overline{v^{2}} S_{d}\left(t, t_{0}\right)=\overline{v^{2}} S_{p}\left(t, t_{0}\right)+\kappa\left(t-t_{0}\right) \overline{v\left(t_{0}\right)\left(\nabla^{2} u_{2}\right)_{p}}\left[1+O\left\{\omega\left(t-t_{0}\right)\right\}\right]
$$

The second term in (3.6) arises from the interaction, but it involves what is essentially a Lagrangian-Eulerian correlation. However, if $t-t_{0}$ is small compared with $\omega^{-1}$, then

$$
\overline{v\left(t_{0}\right)\left(\nabla^{2} u_{2}\right)_{p}} \doteqdot \overline{u_{2} \nabla^{2} u_{2}}
$$

since $\nabla^{2} u_{2}$ is determined by the small-scale components of the turbulence which have a time scale $\omega^{-1}$, and the time scale of $v(t)$ will be that of the energy containing eddies. Moreover,

$$
\overline{u_{2} \nabla^{2} u_{2}}=\overline{\nabla \cdot\left(u_{2} \nabla u_{2}\right)}-\overline{\left(\nabla u_{2}\right)^{2}}
$$

where the first term is zero when the small-scale motion is homogeneous, and

if it is also isotropic.

$$
\overline{\left(\nabla u_{2}\right)^{2}}=\frac{1}{3} \omega^{2}
$$

It now follows from (3.6) that,

and substitution in (2.7) then gives

$$
S_{d}(\tau)=S_{p}(\tau)-\frac{1}{3} \frac{\kappa \omega^{2}}{\bar{v}_{2}} \tau+O\left(\tau^{2}\right)
$$

$$
D^{2}=\overline{Y^{2}}+2 \kappa\left(t-t_{0}\right)-\frac{1}{9} \kappa \omega^{2}\left(t-t_{0}\right)^{3}
$$

correct to order $\left(t-t_{0}\right)^{3}$. The initial effect of the interaction between the turbulence and the molecular diffusion is thus to reduce the dispersion relative to the origin.*

In view of the fact that this conclusion differs from that reached previously, it is perhaps worthwhile repeating qualitatively the present argument. An element

* Since we are concerned here with the initial stages of the diffusion, it would in fact suffice for the present argument if only the small-scale components of the turbulence were stationary and isotropic. 
of substance spreads initially due to molecular diffusion like $\delta=\left\{\kappa\left(t-t_{0}\right)\right\}^{\frac{1}{2}}$. The order of magnitude of the difference between the velocities with which different parts of the element are convected is $\delta\left(\partial u_{2} / \partial x\right)+\frac{1}{2} \delta^{2}\left(\partial^{2} u_{2} / \partial x^{2}\right)+$ smaller terms. For homogeneous turbulence, the covariance of $u_{2}$ and $\partial u_{2} / \partial x$ is zero, but that of $u_{2}$ and $\partial^{2} u_{2} / \partial x^{2}$ is negative. Thus on average, the speed at which the substance is convected away from the source is less than that of the (originally coincident) fluid particle, and the dispersion is thereby reduced.

Another way of phrasing the argument is to note that from (3.8) $S_{d}(\tau)<S_{p}(\tau)$, at least initially. That is, the effect of molecular diffusion is, so to speak, to make the molecules of the diffusing substance forget more quickly than infinitesimal fluid elements what their original velocity of convection was. Since we should intuitively expect this effect to become more marked as $\tau$ increases, we conclude that $S_{d} / S_{p}$ is a decreasing function of $\tau$, and that $\Delta$ continues to decrease as $t-t_{0}$ increases.* We shall return to this point in $\$ 5$.

It remains to discuss why the results given here differ from those obtained in I by a somewhat different analysis. Light is thrown on this by consideration of the dispersion of a small amount of the substance released instantaneously, i.e. a spot.

\section{Dispersion of a spot}

A corollary of the present argument is that the centroid of the spot will lag behind, on average, the fluid particle released at the same instant. To calculate by how much, we multiply (2.8) by $y$ and integrate over all space, obtaining

$$
\frac{d}{d t} \int\left(y-y_{p}\right) \theta d \mathbf{x}=\int\left(u_{2}-v\right) \theta d \mathbf{x},
$$

where $y_{p}$ and $v$ are the displacement and velocity of the fluid particle. To the same order of approximation as in $\S 3$, the right-hand side of (4.1) can be evaluated to give

$$
\int\left(u_{2}-v\right) \theta d \mathbf{x}=\kappa\left(t-t_{0}\right)\left(\nabla^{2} u_{2}\right)_{p}
$$

The left-hand side of (4.1) is the rate of change of the distance (in the $y$-direction) between the centroid of the heat spot and the fluid particle. The displacement of the centroid relative to the origin is therefore $Y+\frac{1}{2} \kappa\left(t-t_{0}\right)^{2}\left(\nabla^{2} u_{2}\right)_{p}$, correct to order $\left(t-t_{0}\right)^{2}$. Since $Y \doteqdot v\left(t_{0}\right)\left(t-t_{0}\right)$ for small values of $t-t_{0}$, it follows that the dispersion of the centroid is, relative to the point of release,

$$
\overline{Y^{2}}-\frac{1}{3} \kappa \omega^{2}\left(t-t_{0}\right)^{3},
$$

where $\overline{Y^{2}}$ is the dispersion of the fluid particle.

Moreover, it is easy to calculate the shape of the spot for small values of $t-t_{0}$ (see Townsend 1951), and it follows from his results that the dispersion in a given direction of the heat spot relative to its centroid is

$$
2 \kappa\left(t-t_{0}\right)+\frac{4}{9} \kappa\left(t-t_{0}\right)^{3}\left(\overline{\alpha^{2}}+\overline{\beta^{2}}+\overline{\gamma^{2}}\right),
$$

* A very similar argument was put forward independently by Dr T. H. Ellison in a private communication in 1956 . 
where $\alpha, \beta$ and $\gamma$ are the principal rates of strain, and $\overline{\alpha^{2}}+\overline{\beta^{2}}+\overline{\gamma^{2}}=\frac{1}{2} \omega^{2}$. Notice that the interaction term in (4.4) is positive, so that the interaction increases the dispersion of the spot relative to its centroid and, as a consequence, increases the rate at which the maximum concentration in the spot decreases, as was verified experimentally by Townsend (1951). However, combining (4.4) and (4.3), we find that the dispersion of the spot relative to the point of release is given by (3.9). That is to say, although the dispersion of the spot relative to its centroid is increased by the interaction, the dispersion of the centroid of the spot is reduced by a greater amount so that the total dispersion relative to the point of release is reduced.

We can now account for the difference between the present result and that given previously in I. In that work, the width of the contorted diffusing wake behind a continuous source moving (rapidly) through a turbulent velocity field was calculated, and it was shown that the effect of the interaction is to make this greater than $2 \kappa\left(t-t_{0}\right)$. The present work in no way contradicts this result. However, it was tacitly assumed in the previous work that the instantaneous axis of the diffusing wake coincides with the locus of the fluid particles coming from the source. The present work shows that this assumption is unjustified, and that on average the displacement of the instantaneous axis is less than that of the fluid particles by an amount which more than compensates for the increase in the instantaneous wake width due to the interaction.

\section{The interaction for large times}

All the available evidence indicates strongly that $\overline{Y^{2}} \propto\left(t-t_{0}\right)$ when $t-t_{0}$ is comparable with $t_{e}$, a time characteristic of the energy containing eddies, and that we can write

$$
\overline{Y^{2}} \sim C \overline{v^{2}} t_{e}\left(t-t_{0}\right),
$$

where $C$ is a constant of order unity. (For definiteness, we may suppose $t_{e}$ is given by $\nu \omega^{2} t_{e} / \overline{v^{2}}=\frac{3}{2}$; then for the decaying homogeneous turbulence downstream of a grid $t_{e}=t_{0}$, where $t_{0}$ is the time from a vertical origin at which the energy of the turbulence is infinite.) The expression (3.9) can be written

$$
D^{2}=\overline{Y^{2}}+2 \kappa\left(t-t_{0}\right)-\frac{\kappa}{v} \overrightarrow{v^{2}} \frac{\left(t-t_{0}\right)^{3}}{t_{e}}
$$

and is therefore clearly of limited value since it becomes negative as $t-t_{0}$ increases. In fact, (5.2) is unlikely to hold far outside its theoretical range of validity as given by (1.5). This is in practice so small (see I) that it is desirable to make an estimate of $D^{2}$ for larger values of $t-t_{0}$. The following discussion is intuitive and somewhat speculative, but seems worth giving in the absence of anything better, and the conclusions should be amenable to experimental investigation.

We still consider stationary, homogeneous turbulence, although later we shall apply the conclusions to cover decaying homogeneous turbulence behind a grid. The general argument presented at the end of $\S 3$ shows that $S_{p}(\tau)>S_{d}(\tau)$, and moreover the argument implies that $S_{p} / S_{d}$ is an increasing function of $\tau$. Now this ratio is determined primarily by an interaction between the molecular diffusion and the small-scale straining motions, so that when $\tau \gg \omega^{-1}$ the interaction will 
cease to have any further effect and $S_{p} / S_{d}$ should tend to a value independent of $\tau$. We can express this argument formally as follows.

It follows from (2.10) that

$$
S_{d}(\tau)-S_{p}(\tau)=\left(\overline{v^{2}}\right)^{-1} \int \overline{\theta\left\{u_{2}-v(t)\right\} v\left(t_{0}\right)} d \mathbf{x},
$$

where $\tau=t-t_{0}$. Now the value of $\theta\left\{u_{2}-v(t)\right\}$ is determined mainly by the smallscale motion, whereas $v(t)$ is determined by the energy containing eddies, which suggests that

$$
\overline{\theta\left\{u_{2}-v(t)\right\} v\left(t_{0}\right)} \doteqdot S_{p}(\tau) \overline{\theta\left\{u_{2}-v(t)\right\} v(t)}
$$

when the Reynolds number of the turbulence is sufficiently large, where the term with the overbar is determined mainly by the molecular diffusivity, the rate of strain, and $\tau$. Hence, from dimensional considerations,

$$
S_{d}(\tau)-S_{p}(\tau)=-\left(\overline{v^{2}}\right)^{-1} S_{p}(\tau) \kappa \omega f(\omega \tau)
$$

where $f(\omega \tau)$ is a positive increasing function of $\tau$ to express the idea that the interaction continues to grow with $\tau$. When $\tau \gg \omega^{-1}, f(\omega \tau)$ either tends to a constant or infinity; the latter is impossible since it would make $D^{2}$ negative, so we come to the conclusion that

$$
S_{d}(\tau)-S_{p}(\tau)=-a \kappa \omega\left(\overline{v^{2}}\right)^{-1} S_{p}(\tau)
$$

when $\omega \tau \gg 1$, where $a$ is a (positive) constant, of order unity, depending on the structure of the turbulence.

The relation (5.5) suggests that an appropriate expression valid for all $\tau$ is

$$
S_{d}(\tau)-S_{p}(\tau)=-a \kappa \omega\left(\overline{v^{2}}\right)^{-1} S_{p}(\tau)\left\{1-e^{-b \omega \tau}\right\}
$$

which agrees with (3.8) for $\omega \tau \ll 1$ if $a b=\frac{1}{3}$, and reduces to (5.5) when $\omega \tau \gg 1$.

The derivation of (5.5) or (5.6) is intuitive and far from watertight, but the argument is supported by the fact that these expressions have the properties which we should intuitively associate with $S_{d}-S_{p}$; e.g. the contribution to the interaction from the energy containing eddies is independent of the contribution from the small-scale motion, and the greater $\kappa$ and $\omega$, the greater the interaction. (Moreover, multiplying (5.5) or (5.6) by any function with a time scale comparable with $t_{e}$, to take account of any possible effect of the energy containing eddies on the interaction in addition to that represented by $S_{p}(\tau)$, can be shown to have insignificant effect.)

It now follows from substitution into (2.7) that, for $\omega\left(t-t_{0}\right) \geqslant 1$,

$$
\Delta=-a \kappa \omega \frac{\overline{Y^{2}}}{\overline{v^{2}}}=-a \sqrt{ } 15 \frac{\kappa}{v} R_{\lambda}^{-1} \overline{Y^{2}}
$$

where $R_{\lambda}$ is the Reynolds number based on $\left(\overline{v_{2}}\right)^{\frac{1}{2}}$ and the dissipation length parameter $\lambda=\sqrt{ }\left(15 \overline{v^{2}} / \omega^{2}\right)$. Thus $\Delta / \overline{Y^{2}}$ is inversely proportional to the Prandtl number and $R_{\lambda}$. The ratio of $|\Delta|$ and the direct effect of molecular diffusion is

$$
\frac{|\Delta|}{2 \kappa\left(t-t_{0}\right)}=\frac{2}{4 \sqrt{ } 15} a C R_{\lambda}
$$

where we have used (5.1) for $\overline{Y^{2}}$. Thus if the Reynolds number of the turbulence is sufficiently large, the direct effect of molecular diffusion is small compared with the interaction term. 
For decaying homogeneous turbulence downstream of a grid, it is known that $R_{\lambda}$ is constant during the initial period of decay, and that

$$
R_{\lambda}^{2}=\frac{10}{A} \frac{U M}{\nu},
$$

where $U$ is the mean velocity, $M$ is the spacing length of the grid, and $A$ is a constant depending on the grid shape $(A=135$ for the grids employed by Mickelsen 1960). We can therefore obtain an estimate of $a$ from the data presented in figure 2 of his paper. Taking a rough mean of his measurements for

$$
\left(t-t_{0}\right) / t_{0}>0 \cdot 6
$$

we obtain (neglecting one pair of points which appear inconsistent and which may be due to experimental error)

$$
\begin{aligned}
\frac{\Delta_{\mathrm{CO}_{2}}-\Delta_{\mathrm{He}}}{\overline{Y^{2}}} & \doteqdot 0.18 \text { for } \quad R_{\lambda}=20 \\
& \doteqdot 0.07 \text { for } \quad R_{\lambda}=40,
\end{aligned}
$$

whereas (5.7)predicts that this ratio should be $13 \cdot 5 a / R_{\lambda}$. Theexperimental measurements are therefore in rough quantitative agreement if $a=0 \cdot 23$. Incidentally, for these comparatively low values of $R_{\lambda}$, the interaction and the direct effect of molecular diffusion are of the same order of magnitude.

\section{Appendix}

We shall now discuss the covariances $\overline{\langle q(t) V(t+\tau)}\rangle$ and $\overline{\langle q(t+\tau) V(\bar{t})}\rangle$, which are functions of $\tau$. Let $p\left(\mathbf{x}, \mathbf{q}, t \mid \mathbf{x}_{0}, t_{0}\right)$ denote the probability, for $t-t_{0} \gg \tau_{c}$ and for one realization of the turbulence, that a marked molecule of the substance is in the element of phase space $d \mathbf{x} d \mathbf{q}$, given that it was at $\mathbf{x}_{0}$ at time $t_{0}$ with Brownian velocity $q_{0}$, where the function $p$ is a random variable over the ensemble of the possible turbulent motions. The assumption of the independence of the Brownian and macroscopic velocities is here made use of explicitly by saying that $p$ is independent of $\mathbf{q}_{0}$ when $t-t_{0} \gg \tau_{c}$. We have

$$
\theta\left(\mathbf{x}, t \mid \mathbf{x}_{0}, t_{0}\right)=\int p\left(\mathbf{x}, \mathbf{q}, t \mid \mathbf{x}_{0}, t_{0}\right) d \mathbf{q},
$$

where $\theta$ is the solution of the diffusion equation (2.8), and moreover

$$
\int q_{i} p\left(\mathbf{x}, \mathbf{q}, t \mid \mathbf{x}_{0}, t_{0}\right) d \mathbf{q}=-\kappa \frac{\partial \theta}{\partial x_{i}}\left(\mathbf{x}, t \mid \mathbf{x}_{0}, t_{0}\right)
$$

where the suffix $i$ denotes the component in the $i$-direction.

If $f$ is any function of the marked molecule,

and

$$
\begin{gathered}
\langle f\rangle=\iint f p d \mathbf{x} d \mathbf{q}, \\
\langle\bar{f}\rangle=\lim _{V \rightarrow \infty} \frac{1}{\bar{V}} \int_{V}\langle f\rangle d \mathbf{x}_{0},
\end{gathered}
$$

since the turbulence is homogeneous and an average over the turbulent ensemble is equivalent to integrating with respect to $\mathbf{x}_{0}$ over a large region of space. 
It now follows that

and that

$$
\begin{gathered}
\left\langle q_{i}(t)\right\rangle=-\kappa \int \frac{\partial \theta}{\partial x_{i}} d \mathbf{x}=0, \\
\left\langle\overline{\left.q_{i}(t)\right\rangle}=-\overline{\left\langle V_{i}(t)\right.}\right\rangle=0 .
\end{gathered}
$$

The covariances are given by (no summation implied and writing $t^{\prime}=t+\tau$ )

$$
\begin{aligned}
\left\langle q_{i}(t) V_{i}(t+\tau)\right\rangle & =\iint\left[\iint u_{i}\left(\mathbf{x}^{\prime}, t^{\prime}\right) p\left(\mathbf{x}^{\prime}, \mathbf{q}^{\prime}, t^{\prime} \mid \mathbf{x}, t\right) d \mathbf{x}^{\prime} d \mathbf{q}^{\prime}\right] q_{i} p\left(\mathbf{x}, \mathbf{q}, t \mid \mathbf{x}_{0}, t_{0}\right) d \mathbf{x} d \mathbf{q} \\
& =-\kappa \iint u_{i}\left(\mathbf{x}^{\prime}, t^{\prime}\right) \theta\left(\mathbf{x}^{\prime}, t^{\prime} \mid \mathbf{x}, t\right) \frac{\partial}{\partial x_{i}} \theta\left(\mathbf{x}, t \mid \mathbf{x}_{0}, t_{0}\right) d \mathbf{x} d \mathbf{x}^{\prime}
\end{aligned}
$$

and

$$
\begin{aligned}
\left\langle q_{i}(t+\tau) V_{i}(t)\right\rangle & \left.=\iiint \iint q_{i}^{\prime} p\left(\mathbf{x}^{\prime}, \mathbf{q}^{\prime}, t^{\prime} \mid \mathbf{x}, t\right) d \mathbf{x}^{\prime} d \mathbf{q}^{\prime}\right] u_{i}(\mathbf{x}, t) p\left(\mathbf{x}, \mathbf{q}, t \mid \mathbf{x}_{0}, t_{0}\right) d \mathbf{x} d \mathbf{q} \\
& =-\kappa \iint \frac{\partial}{\partial x_{i}^{\prime}} \theta\left(\mathbf{x}^{\prime}, t^{\prime} \mid \mathbf{x}, t\right) u_{i}(\mathbf{x}, t) \theta\left(\mathbf{x}, t \mid \mathbf{x}_{0}, t_{0}\right) d \mathbf{x} d \mathbf{x}^{\prime},
\end{aligned}
$$

where we have made use of (A 1) and (A 2). We note that (A 5) is zero, since the integral with respect to $\mathrm{x}^{\prime}$ vanishes, but that (A 4) is not in general zero for one particular realization of the turbulence. (The physical interpretation of this result is that, by (A 2), the value of $q_{i}$ at a point $\mathbf{x}$ is correlated with $\mathbf{x}$ if the molecule has come from $x_{0}$, and the continuum velocity at $\mathbf{x}^{\prime}$ is also correlated with $\mathbf{x}$.) However, the average of (A 4) over the turbulent ensemble vanishes since

$$
\int \frac{\partial}{\partial x_{i}} \theta\left(\mathbf{x}, t \mid \mathbf{x}_{0}, t_{0}\right) d \mathbf{x}_{0}=\mathbf{0}
$$

Hence (dropping the suffix $i$ )

$$
\langle\overline{q(t) \overline{V(t+\tau)}}\rangle=\langle\overline{q(t+\tau) \overline{V(t)}\rangle}=0,
$$

provided, of course, $\tau \gg \tau_{c}$.

If this last condition is not satisfied, the covariances will not in general vanish. We can obtain an estimate of this effect as follows. The covariance does not vanish in a time of order $\tau_{c}$ because the molecule still remembers, so to speak, that it has come from a region where the fluid velocity differed by an amount of order $\lambda \omega$, where $\lambda$ is the mean free path. (It is only differences in the fluid velocity that matter.) Thus,

$$
\left\langle\overline{V(t) q(t+\tau)\rangle}=\lambda \omega \vec{q} f\left(\tau / \tau_{c}\right)\right.
$$

where $\bar{q}$ is the root-mean-square Brownian velocity and $f$ is dimensionless, and similarly for the other covariance. A contribution of this type to $\mathfrak{S}(\tau)$ gives a contribution to $D^{2}$ of amount

$$
2 \lambda \omega \bar{q} \tau_{c}\left(t-t_{0}\right) \int_{0}^{\infty} f(\xi) d \xi
$$

when $t-t_{0} \gg \tau_{c}$. Remembering that $\lambda \bar{q} / \kappa=O(1)$, we see that this is negligible compared with $2 \kappa\left(t-t_{0}\right)$, the direct effect of molecular diffusion. Similarly, the difference between $\langle q(t) q(t+\tau)\rangle$ and the value it would have in a fluid at rest gives rise to an effect of the same order of magnitude. 
Finally, a comment seems appropriate on the difficulty of deriving the expression (2.7) from formal manipulation of the diffusion equation. The point seems to be that the $\theta$ in (2.10) is really a probability, whereas the $\theta$ in the diffusion equation is a concentration. The two are the same only if $\omega \tau_{c} \ll 1$, and so it appears that formal manipulation of the diffusion equation, without explicit use in some way (not yet clear) of the condition $\omega \tau_{c} \ll 1$, will not give (2.7).

I wish to thank Dr T. H. Ellison for the benefit of a lengthy correspondence, in which a fallacy in earlier arguments, in particular the non-vanishing of the covariance $\langle q(t) V(t+\tau)\rangle$, was made clear to me.

\section{REFERENCES}

Batchelor, G. K. \& Townswid, A. A. 1956 Article in Surveys in Mechanics, p. 352. Cambridge University Press.

MickmLSEN, W. R. 1960 J. Fluid Mech. 7, 397.

Taylor, G. I. 1921 Proc. Lond. Math. Soc. 20, I96.

Townsend, A. A. 1951 Proc. Roy. Soc. A, 209, 418.

Townsend, A. A. 1954 Proc. Roy. Soc. A, 224, 487. 